\title{
Electromagnetic Field Simulation in a Microwave Chamber with Multiple Waveguides
}

\author{
Jaekyung $\mathrm{Kim}^{1}$ and Euysik Jeon ${ }^{2, a}$ \\ ${ }^{1}$ Department of Mechanical Engineering, Kongju National University College of Engineering, Korea \\ ${ }^{2}$ Department of Mechanical Engineering, Industrial Technology Research Institute, Kongju National University \\ College of Engineering, Korea
}

\begin{abstract}
While microwaves have many features and advantages, problems may occur, including non-heating, partial overheating, and fire due to damaged magnetrons caused by reflected waves, when they are used without a proper understanding of the permittivity of the object to be heated, the electromagnetic field distribution, the matching between the chamber and the waveguide, and the reflected electromagnetic waves. Simulation was performed using the Ansys HFSS tool. Conditions for the uniform electromagnetic field were derived using the distance from the waveguides to the ceramic material as well as the microwave energy intensity as major parameters.
\end{abstract}

\section{Introduction}

Microwaves are powerful energy sources with excellent reproducibility that can be applied to many applications. Microwave energy has been applied in a variety of industries. It has reduced work hours and improved product quality by using uniform heating. [1]

In addition to the four frequency bands used in industrial, scientific, and medical applications, 2.45 $\mathrm{GHz}$ is widely used because it has an appropriate penetration thickness to penetrate laboratory reaction capacity and there are energy sources that can produce microwaves in this frequency band. [2]

In the case of ceramic processes including water, a significantly fast drying speed can be achieved and the non-uniformity of humidity, depending on the position, can be improved using microwaves because the temperature distribution is opposite to that of the existing method.

In addition, when microwaves are used for the burning of ceramic, the temperature rises from the inside of the material. Therefore, if microwaves and surface heating technology are combined, a very uniform temperature distribution can be obtained.

As the reaction between microwaves and materials can heat only specific parts, such as the interface, the application of microwaves to bonding processes can yield faster and better performance than the existing method (joining) and can control the characteristics of composite materials at the interface.

While microwaves have many features and advantages, problems may occur, including nonheating, partial overheating, and fire due to damaged magnetrons caused by reflected waves, when

\footnotetext{
${ }^{\mathrm{a}}$ Corresponding author: osjun@kongju.ac.kr
} 
they are used without a proper understanding of the permittivity of the object to be heated, the electromagnetic field distribution, the matching between the chamber and the waveguide, and the reflected electromagnetic waves.[3] As such, many studies have been conducted on the uniformity of an electromagnetic field to avoid hot spots that could damage magnetrons or destroy the material to be heated. It has been reported that various materials, including zirconia, alumina, and nylon, can be damaged by heat concentration. [4-5]

The penetration depth of microwaves into a material is dependent on their electrical characteristics, and heat is generated accordingly. Electromagnetic wave absorption causes macroscopic, microscopic, and structural changes in the material shape, and the electric field distribution is a major element that affects the process of heating (processing) the material in a microwave chamber.

This study analyzed and determined the installation locations of the waveguides through which microwaves are introduced into a chamber by analyzing the characteristics of microwaves and the electromagnetic field. Simulation was performed using the Ansys HFSS tool. Conditions for the uniform electromagnetic field were derived using the distance from the waveguides to the ceramic material as well as the microwave energy intensity as major parameters.

\section{A microwave chamber with six waveguides}

The microwave simulation chamber was $400 \mathrm{~mm} \times 400 \mathrm{~mm} \times 192 \mathrm{~mm}$ in size and made of stainless steel. WR-340 was used for the waveguides in accordance with the international standard. The microwave input power was $1 \mathrm{~kW}$ and the frequency was $2.45 \mathrm{GHz}$, while the heating time was set as a parameter. As the initial simulation settings, the microwave input and physical properties of the ceramic in the chamber were selected.

The typical heating temperature of ceramic materials is between $500{ }^{\circ} \mathrm{C}$ and $1200{ }^{\circ} \mathrm{C}$. The heating temperature required in this study was $700{ }^{\circ} \mathrm{C}$. As heating time can be shortened by the microwave input power, the maximum number of the waveguides was set to six $(6 \mathrm{~kW})$. Furthermore, the waveguides were placed symmetrically at constant intervals, considering the interference and influence among them. Figure 1 shows schematic diagram modelled microwave chamber and table 1 shows main properties of materials.

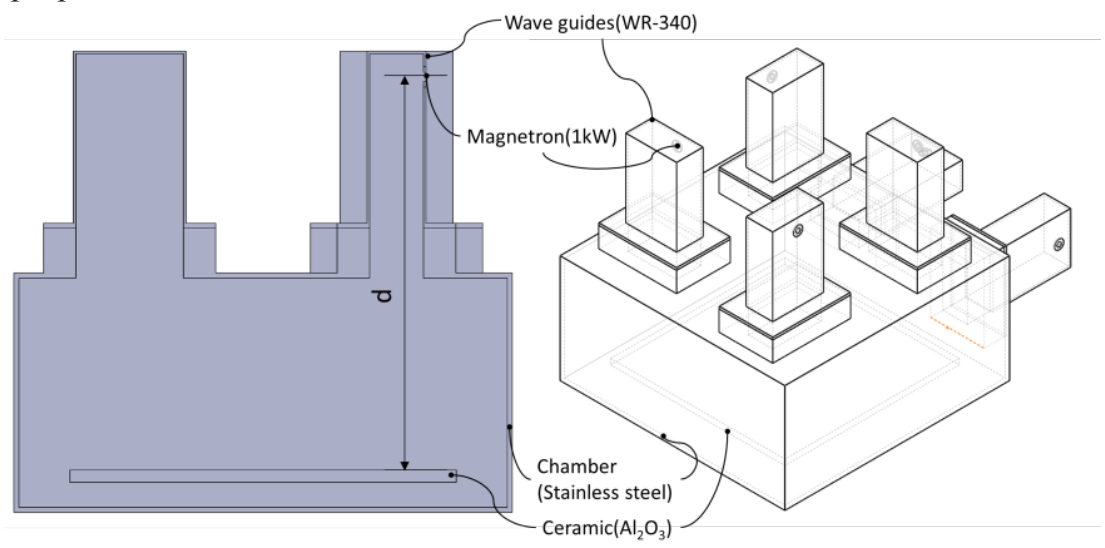

Figure 1. Schematic diagram modelled microwave chamber

Table 1. The material's characteristics

\begin{tabular}{|c|c|c|}
\hline Materials & Stainless steel & Ceramic \\
\hline Relative permittivity & 1 & 9.8 \\
\hline
\end{tabular}




\begin{tabular}{|c|c|c|}
\hline Relative permeability & 1 & 1 \\
\hline Thermal conductivity(w/mk) & 13.8 & 35 \\
\hline
\end{tabular}

\section{Simulation results}

While the frequency change of magnetrons is very important for the electric field distribution inside the chamber, a small change of $10 \%$ occurs and the frequency of the electric field increases mainly due to the wavelength decrease as the frequency increases [6]. Therefore, the simulation frequency was fixed at $2.45 \mathrm{GHz}$.

\subsection{Electric field distribution by Input power}

The electric field distribution was simulated using the six installed waveguides according to the microwave intensity $(1-6 \mathrm{~kW})$. Simulation results showed that the microwave intensity affected the electric field distribution and that higher microwave intensity led to better electric field uniformity. This appears to be influenced by the symmetrical locations of the waveguides. Figure 2 shows electric field distribution by input power.

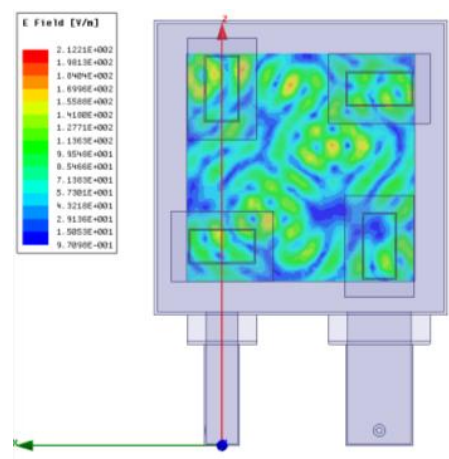

(a) $1 \mathrm{~kW}$

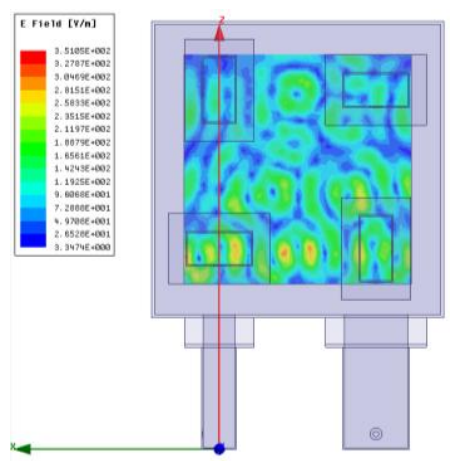

(b) $2 \mathrm{~kW}$

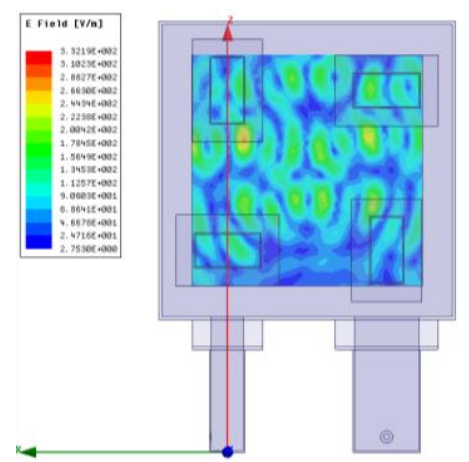

(c) $3 \mathrm{~kW}$

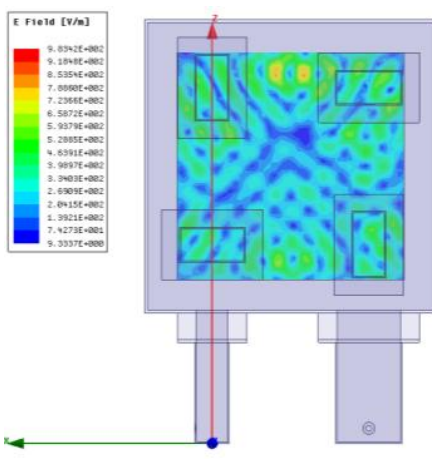

(d) $4 \mathrm{~kW}$

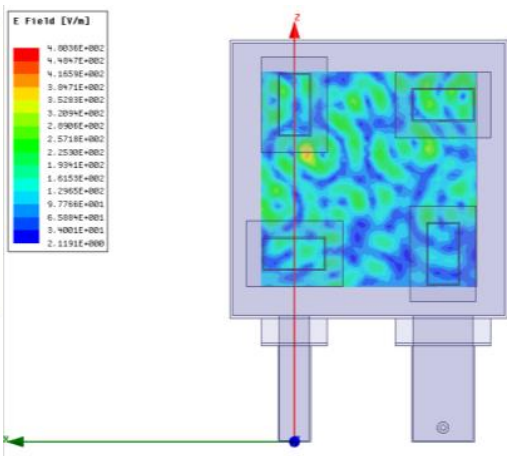

(e) $5 \mathrm{~kW}$

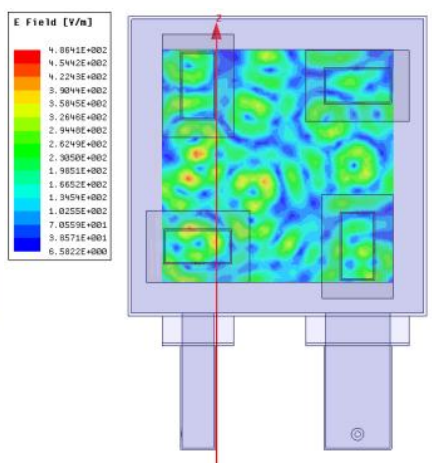

(f) $6 \mathrm{~kW}$

Figure 2. Electric field distribution by input power 
Simulation was conducted to investigate the electric field distribution according to the distance from the multiple waveguides to the ceramic material. As a result, it was confirmed that changes in the distance from the magnetrons to the ceramic material affected the electric field distribution.

The distance between the magnetron and the ceramic material was set as a ratio. When the distance is $60 \%$ and $80 \%$, the deviation value of the E-field is small. Such deviations can cause temperature imbalance or temperature concentration. The current ceramic thickness was set to $10 \mathrm{~mm}$, but it is expected that the electric field distribution will be changed according to the size and shape of the material. Therefore, it is considered that the optimal locations must be derived for each experiment according to the chamber size and material shape. Figure 3 show electric field distribution and simulations results by distance between ceramic and magnetron.

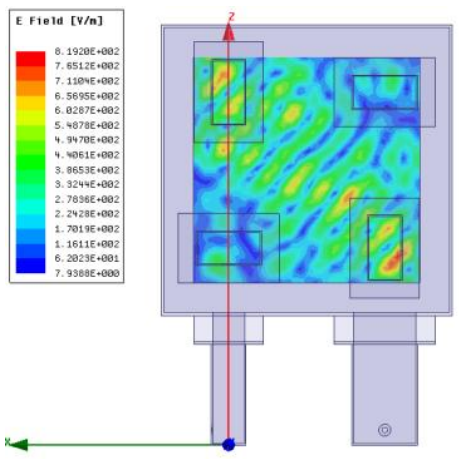

(a) $30 \%$

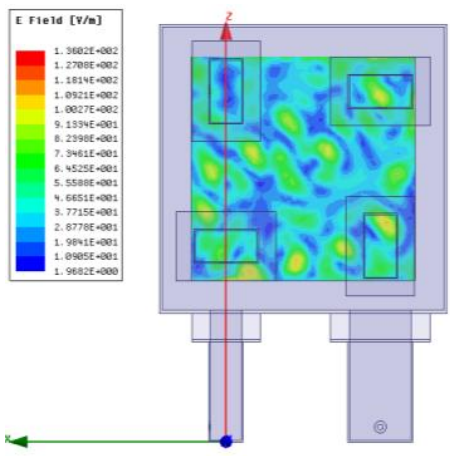

(b) $60 \%$

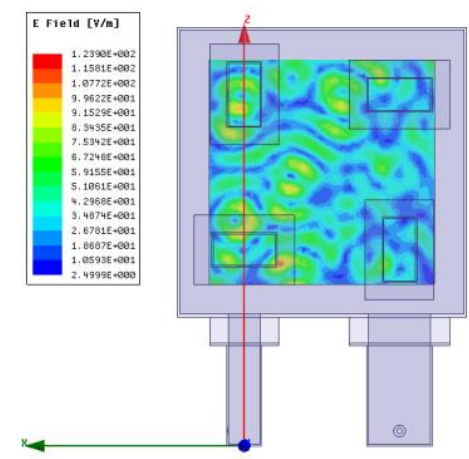

(c) $80 \%$

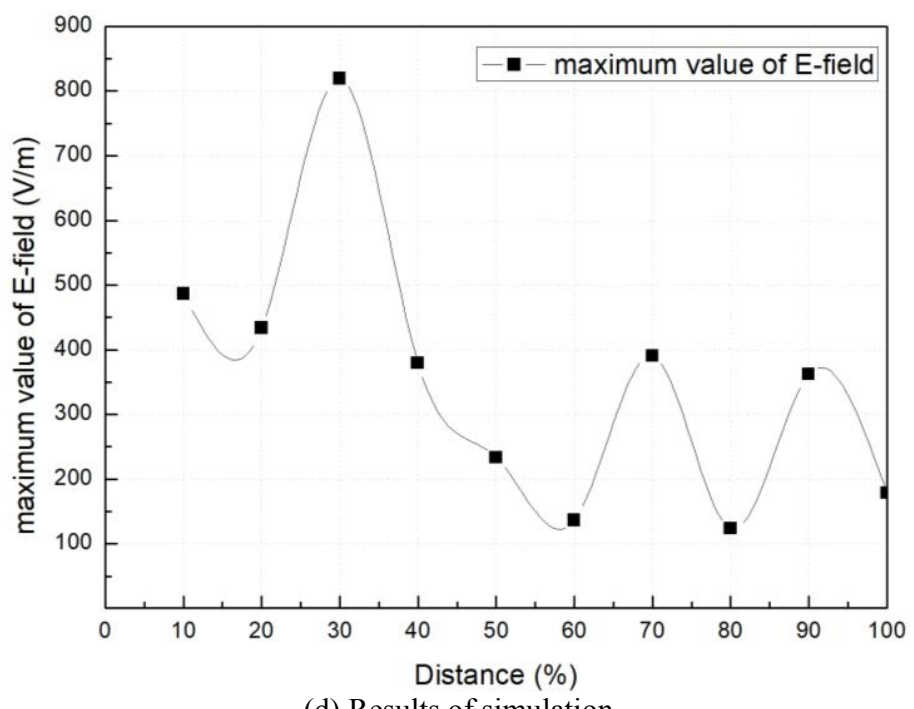

(d) Results of simulation

Figure 3. Simulation results by distance between ceramic and magnetron

\section{Conclusions}

This study conducted an electric field analysis on a shielded chamber with multiple waveguides using ANSYS HFSS software. The effect of the microwave input power on the electric field distribution was investigated and it was confirmed that the electric field distribution became more uniform as microwave intensity increased when the magnetrons were symmetrically installed. It was also confirmed that changes in the distance from the magnetrons to the ceramic material affected the 
electric field distribution in the material. Additional simulation will be conducted in the future to investigate the effect of the electric field distribution on the temperature change of the material. Simulation will also be performed on the optimal distance from the ceramic material to the magnetrons and on the electric field distribution according to the patterns of multiple waveguides.

\section{Acknowledgments}

This research was supported by Basic Science Research Program through the National Research Foundation of Korea(NRF) funded by the Ministry of Science and ICT(NRF-2017R1A2B4009929)

\section{References}

1. Che Woo Seong, Jou. of Korean Soc. of Mechanical Technology, 16, 1(2014)

2. Adu, B. And Otten, L., Journal of microwave Power and Electro-magnetic Energy, 31, 1(1993)

3. Han-Ji Ju and Qing Zhao, JOURNAL OF ELECTRONIC SCIENCE AND TECHNOLOGY OF CHINA, 7, 2(2009)

4. M. Arai, J. G. Binner, G. E. Carr, T. E. Cross, Microwave Processing of Materials III, 269,(1992)

5. D. L. Johnson, D. J. Skamser, M. S. Spotz, Ceramic Transactions: Microwave Theory and Applications in Materials Processing II, 36 (1993)

6. T. Santos, L. C. Costa, M. Valente, J. Monteiro, J. Sousa, Excerpt from the Proceedings of the COMSOL Conference (2010) 